\title{
Age Structure, Mating System, and Population Viability
}

\author{
Stéphane Legendre
}

\subsection{Introduction}

The fate of populations depends on the life-history traits of the species and possible adaptive changes in these traits in response to selective pressure. In unstructured population models, life-history traits are compounded into few parameters, like the intrinsic growth rate $r$ and the carrying capacity $K$ (see Chapter 2). Structured population models, based on life-cycle graphs, allow the effects of specific life-history traits (survival rates, fecundities, generation time, age at maturity) on population dynamics to be investigated. For example, sensitivities of the growth rate to changes in life-cycle transitions can be computed. Individual life-history traits are important determinants of a population's extinction risk, and are also both factors in and targets of a population's adaptive response to environmental change.

When population size is small - always a concern in conservation biology both individual life-history traits and the structure of interactions between individuals and the genetic system are expected to influence viability. The mating system, for example, may be conducive to an Allee effect (see Chapter 2), and inbreeding depression is a potentially important factor of the extinction process of small populations. In this chapter, we study the interplay between population structure, in terms of age and sex, and population persistence. Two-sex structured models that incorporate specific features of the social mating system and possible differences in male and female life cycles are analyzed. Also, attempts to merge genetic factors and demography into integrated models are presented. Size-structured models, more appropriate to plants and some animal species, are not considered here, but lead to similar developments.

\subsection{Extinction Risk in Age-structured Populations}

A life-cycle graph is a macroscopic description of an average organism within a population, describing the effects of the life-history traits. A population is considered as a set of individuals that share the same life cycle, and is structured in age classes. The life-history trait and resultant demographic parameters (survival, fecundity) quantify the flows of individuals between age classes. Iterating the life cycle in discrete time realizes the dynamics of the average life-history phenotype in a given environment. 


\section{Essentials about structured deterministic models}

The life cycle translates into matrix form, which enables several demographic quantities to be computed, among which is the long-term growth rate $\lambda$ (Caswell 1989, 2001; Stearns 1992). A matrix $A=\left(a_{i j}\right)$ can be assembled, with $a_{i j}$ being the contribution of an individual in age class $i$ to age class $j$, from one time step to the next. The nonzero entries of the matrix are the demographic parameters. The population trajectory is obtained by iterating over time according to the recursion equation

$$
\vec{N}(t+1)=A \vec{N}(t),
$$

where $\vec{N}(t)=\left(N_{1}(t), \ldots, N_{n}(t)\right)^{T}$ denotes the population vector at time $t$, and $N_{i}(t)$ the number of individuals in age class $i$; there are $n$ age classes. The population size at time $t$ is $N(t)$, the sum of the entries of the population vector. An example of a general female-based matrix $A$ is

$$
A=\left(\begin{array}{cccc}
\sigma \phi_{0} b_{1} & \ldots & \sigma \phi_{0} b_{n-1} & \sigma \phi_{0} b_{n} \\
\phi_{1} & \ldots & 0 & 0 \\
\vdots & \ddots & \vdots & \vdots \\
0 & \ldots & \phi_{n-1} & \phi
\end{array}\right),
$$

with $\phi_{0}$ being the juvenile survival rate, $\phi_{1}, \ldots, \phi_{n-1}$ subadult survival rates, $\phi$ the adult survival rate, and $b_{1}, \ldots, b_{n}$ fecundities. The primary female sex ratio $\sigma$ (proportion of females at birth) is emphasized here as a parameter, the usual value being $\sigma=0.5$. When $\phi=0, A$ takes the form of the so-called Leslie matrix. A prebreeding census is assumed, since juvenile survival rate $\phi_{0}$ appears in matrix $A$ as a multiplicative factor of fecundities in the first row.

The main demographic result is that, after transitory damped oscillations, the population enters a stable regime of exponential growth with rate $\lambda$, whatever the initial population vector $\vec{N}(0)$, where $\lambda$ is the dominant eigenvalue of the matrix $A$. The dynamics of the population depend entirely on the algebraic properties of the matrix. Asymptotically, the population size $N(t)$ is such that $N(t+1) \approx$ $\lambda N(t)$. The celebrated Perron-Frobenius theorem of linear algebra ensures that $\lambda$ is real and positive. The population either increases exponentially $(\lambda>1)$, which results in demographic explosion, or declines exponentially $(\lambda<1)$, which results in population extinction. The degenerate case $\lambda=1$ leads to equilibrium. Convergence toward the asymptotic regime is geometric with rate $1 / \xi$, where $\xi=$ $\lambda /\left|\lambda_{2}\right|$ is the damping ratio, $\lambda_{2}$ being the second eigenvalue of the matrix. The period of the transient oscillations depends mostly on the angle formed by $\lambda_{2}$ and the real axis in the complex plane.

The population structure at time $t$,

$$
\vec{W}(t)=\left(\frac{N_{1}(t)}{N(t)}, \ldots, \frac{N_{n}(t)}{N(t)}\right)^{T},
$$


is the vector of the proportions of individuals in the various age classes. Except for matrixes $A$ with special structure [see Chapter 4 in Caswell (2001) for more details], this vector converges toward a stable population structure $\vec{W}$, known as the stable age distribution. The vector $\vec{W}$ is the right eigenvector of matrix $A$ corresponding to $\lambda$. The left eigenvector $\vec{V}$ of the matrix corresponding to $\lambda$ gives the series of reproductive values indexed by age. These reproductive values describe which age classes contribute most to population size when the asymptotic regime is reached. More precisely, a population's size is asymptotically given by

$$
N(t) \approx \lambda^{t}\langle\vec{V}, \vec{W}(0)\rangle N(0),
$$

where the angular brackets denote the scalar product of vectors. Equation (3.4) yields an estimator of the actual average growth rate until time $t$

$$
\hat{\lambda}=\exp \left(\frac{\ln (N(t))-\ln (N(0))}{t}\right) .
$$

Another important quantity defined at the population level from individual lifehistory traits is the generation time. There are various measures of generation time, one being the mean generation length $\bar{T}$, a weighted sum of the contribution of each age class to offspring once the population has reached its asymptotic regime. For the Leslie matrix, this gives

$$
\bar{T}=\sum_{i=1}^{n} i \Phi(i) \lambda^{-i},
$$

with $\Phi(i)=\sigma \phi_{0} \phi_{1} \ldots \phi_{i-1} b_{i}$.

\section{Factors of population regulation and extinction}

The model described above, based on a constant matrix $A$, can be viewed as a deterministic skeleton upon which density-dependent factors and stochastic processes will operate. Stochastic processes may be endogenous or exogenous: demographic stochasticity and interaction stochasticity pertain to the first kind, whereas environmental stochasticity and catastrophes are of the second kind (see Box 2.1). For small populations, demographic stochasticity, which is unavoidable and strictly dependent on population size, can become the main factor of extinction. The long-term growth rate $\lambda$ of the deterministic skeleton model can always be defined, but stochastic processes generate variation in the instantaneous growth rate and population size. Predictors of the extinction risk, best described by the distribution of time to extinction, typically involve measures of this variation. Usually, to obtain such measures requires intensive computer simulations, but a good deal of mathematical theory is available to guide the simulations and interpret the results (e.g., Ferrière et al. 1996; Mills et al. 1996; Fieberg and Ellner 2001).

Density dependence and stochastic factors that affect the population dynamics can be considered, as a first approximation, to be perturbations of the above model, and their respective influences can be assessed from the sensitivities of the growth rate $\lambda$ to changes in various parameters (Caswell 1989; Tuljapurkar 1990). When 
a parameter $x$ is varied by an amount $\varepsilon$, the growth rate $\lambda$ changes by an amount $\varepsilon s_{\lambda}(x)$, where $s_{\lambda}(x)=\partial \lambda / \partial x$ is the sensitivity of $\lambda$ to changes in $x$. When a parameter $x$ of the model is varied by $\eta \%$, the growth rate $\lambda$ changes by $\eta e_{\lambda}(x) \%$, where $e_{\lambda}(x)=(x / \lambda) s_{\lambda}(x)$ is the elasticity of $\lambda$ to changes in $x$. Thus, elasticity is similar to sensitivity, but it takes the relative magnitude of the parameter change into account. Sensitivities and elasticities enable us to determine those parameters that have the greatest impact on population growth. However, sensitivities only quantify the impact of small independent perturbations (see Mills et al. 1999).

With $\operatorname{Var}\left(a_{i j}\right)$ denoting the variance of temporal fluctuations of the corresponding demographic parameter around its average value $a_{i j}$, the variance in population growth can be approximated by

$$
\operatorname{Var}(\lambda) \approx \sum_{i, j}\left(\frac{\partial \lambda}{\partial a_{i j}}\right)^{2} \operatorname{Var}\left(a_{i j}\right)
$$

Thus, the sensitivities to the demographic parameters act as weights to determine the growth-rate variance from these parameters' variance. The sensitivities are therefore important determinants of the extinction risk. A powerful result from Houllier and Lebreton (1986), which has been little appreciated to date, is the following. With $c$ denoting a common parameter that multiplies the fertilities in the first row of $A$ [for example, $\sigma$ or $\phi_{0}$ in Equation (3.1b)], the elasticity with respect to $c$ is inversely proportional to generation time $\bar{T}$, that is

$$
e_{\lambda}(c)=\frac{c}{\lambda} \frac{\partial \lambda}{\partial c}=\frac{1}{\bar{T}} .
$$

This implies that short-lived species are more sensitive to fluctuations in fertility parameters than long-lived ones. Conversely, long-lived species are, comparatively speaking, more sensitive to fluctuations in the adult survival rate.

Density dependence arises from resource limitation (exogenous, such as food or space, or endogenous, such as partners for reproduction), and results in demographic parameters being functions of the number of individuals in age classes. As competition may involve different resources (food, territory, breeding sites), density dependence may differentially affect the various stages of a life cycle. Negative density dependence leads to extinction or regulation. In the latter case, complex dynamics (quasi-periodicity, chaos) may occur (May and Oster 1976; Ruelle 1989). Notice that a longstanding common wisdom has been that large and unpredictable fluctuations associated with chaotic dynamics should increase a population's vulnerability to extinction. In fact, chaotic population dynamics can result from the operation of natural selection on life-history traits (Ferrière and Gatto 1993), and chaos may create enough asynchrony between local populations connected by migration to promote long-term persistence at the regional scale (Allen et al. 1993). Thus, no simple relationship exists between nonlinear dynamics, the extinction risk, and adaptation (see Chapter 11).

Demographic stochasticity, which stems from the random realization of the life cycle by each individual in the population, is modeled by specifying a multitype 
branching process based on the above matrix model (see Section 3.3). Under demographic stochasticity, a population either goes extinct or grows at an average rate $\lambda$. For $\lambda \leq 1$ extinction occurs with certainty. For $\lambda>1$ the probability of extinction is strictly larger than 0 and strictly smaller than 1 ; it depends on the initial population size and structure, and decreases exponentially with initial population size. More precisely, the probability of extinction at time $t$ is

$$
q_{e}(t)=q_{1}(t)^{N_{1}(0)} \ldots q_{n}(t)^{N_{n}(0)},
$$

where $q_{i}(t)$ is the probability of extinction at time $t$ when the initial population consists of a single individual in age class $i$. The average population structure is unaffected by demographic stochasticity. For $\lambda<1$, conditional on being nonextinct, the probability distribution of population size converges toward a constant distribution, known as the population's quasi-stationary distribution. As a consequence, from one time step to the next, the population goes extinct with probability $1-\lambda$, thus behaving like a single individual with survival probability $\lambda$. The quasi-stationary distribution also exists when the population is regulated by density dependence, or is subject to uncorrelated environmental stochasticity: there is a constant parameter $\beta<1$ such that the population behaves as a single individual with survival rate $\beta$ (Gosselin and Lebreton 2000).

Under environmental stochasticity, demographic parameters may vary independently of each other, or co-vary (e.g., a decrease in survival co-occurs with a decrease in fecundity), with or without temporal autocorrelations (Shaffer 1987; Tuljapurkar 1990; Lande 1993; Chapter 2). The usual effect is to reduce the expected growth rate, compared to the value that the growth rate would assume if all parameters were fixed at their average value. A stationary population structure is not guaranteed. The ultimate probability of extinction is independent of the initial population size, but the average time to extinction increases with the initial population size.

All of the above factors contribute to various extents to the risk of extinction. For example, in their survey on translocations, Griffith et al. (1989) find that the initial population size is a strong predictor of the extinction risk. The probability of extinction decreases as the initial population size increases, and remains constant above some population-size threshold. This is what is expected under the combined effects of demographic and environmental stochasticity. Also, an appreciation of how environmental stochasticity and density dependence combine is crucial to forecasting the dynamics of natural populations accurately (e.g., Leirs et al. 1997).

\subsection{Effect of Sexual Structure on Population Viability}

Demographic models usually describe the dynamics of the female population only. However, for small populations, random fluctuations in sex ratio and pair formation may affect persistence (see Chapter 2). To account for the impact of sexual reproduction on population viability, two-sex life cycles with interactions between 
sexes must be constructed. The pattern of pair formation (the social mating system), appears to play an important role in extinction processes: for small populations, the shortage of mates can generate an Allee effect because the female population forms a limiting resource (Chapter 2; Courchamp et al. 1999; Legendre et al. 1999; Stephens and Sutherland 1999; Stephens et al. 1999; Møller and Legendre 2001).

\section{Deterministic two-sex models}

Models of two-sex life cycles can be constructed by duplicating the life-cycle graph for males and females. Reproductive transitions from the female part of the graph connect to both male and female parts; the relative contribution to each part is measured by the primary female sex ratio $\sigma$, which means that female offspring are produced in proportion $\sigma$ and males in proportion $1-\sigma$.

In two-sex models, reproduction parameters become dependent upon the number of mating pairs that can form. Therefore, the mating system ought to be specified (Box 3.1) in terms of a "marriage function" or "mating function" (Caswell and Weeks 1986; see also Heino et al. 1998). This mating function gives the number $M\left(N_{m}, N_{f}\right)$ of matings as a function of the numbers $N_{m}$ and $N_{f}$ of reproductive males and females. Mating models can be designed to account for fidelity, probability of encounter, and asymmetric preferences in males and females depending on social status or age (Gerritsen 1980; Gimelfarb 1988; Castillo-Chavez and Hsu Schmitz 1997). Considering only matings that yield offspring, we assume here that the number of matings is less than the number of sexually mature females, i.e., $M\left(N_{m}, N_{f}\right) \leq N_{f}$.

The mathematical properties of the mating function reflect the structure of encounters between sexes (Caswell and Weeks 1986; Martcheva 1999). One important property is homogeneity, which is the fact that, for any $c \geq 0$, one has $M\left(c N_{m}, c N_{f}\right)=c M\left(N_{m}, N_{f}\right)$. If we define the breeding sex ratio $\rho$ as the proportion of reproductive females in the reproductive population, by virtue of the previous homogeneity property, we have

$$
\frac{M\left(N_{m}, N_{f}\right)}{N_{f}+N_{m}}=M\left(\frac{N_{m}}{N_{f}+N_{m}}, \frac{N_{f}}{N_{f}+N_{m}}\right)=M(1-\rho, \rho) .
$$

Investigating the dynamics of two-sex models is greatly facilitated by considering the following limit function $\mu_{M}$, which is associated with the mating function $M$ for each value of $\sigma$ in $[0,1]$, and is given by

$$
\mu_{M}(\sigma)=\lim _{\rho \rightarrow \sigma} \frac{M\left(N_{m}, N_{f}\right)}{N_{f}+N_{m}}=M(1-\sigma, \sigma) .
$$

The limit function $\mu_{M}$ captures the main features of the mating system. It is 0 for $\sigma=0$ and $\sigma=1$, and is usually concave with a single maximum (Figure 3.1). Furthermore, for $M\left(N_{m}, N_{f}\right) \leq N_{f}$, Equation (3.9b) leads to $\mu_{M}(\sigma) \leq \sigma$, which entails that the graph of the limit function $\mu_{M}$ lies entirely below the main diagonal. Notice that the main diagonal coincides with the graph of the limit function $\mu_{M}$ recovered when the number of mating paris is merely equal to the number of 
Box 3.1 Social mating systems

Several aspects of sexual selection are determined by the social mating system, that is, the way males and females pair for reproduction (Orians 1969; Wade and Arnold 1980; Arnold and Duvall 1994). There are four important mating systems in animals:

Monogamy. A single male and a single female form a strong pair bond, usually involving parental care by both partners. The monogamous mating system is very common in birds (90\% of all bird species). However, extra-pair copulations are frequent and can lead to strong sexual selection (Møller and Birkhead 1994).

Polygyny. A single male mates with several females, who mate only with him. Often, males provide nothing but gametes (e.g., lekking species), or they provide little parental care, but rather territory or protection. Sexual dimorphism is often correlated with the degree of polygyny (pinnipeds, ungulates), an extreme example being that of elephant seals, where males outweigh females by more than five times. Harem sizes are typically 50 individuals, and a large proportion of males never reproduce. Nevertheless, several monogamous species are highly dimorphic. There are also examples of species that switch between monogamy and polygyny according to environment (e.g., Höglund 1996). Monogamy and polygyny could coexist as alternative tactics (Pinxten and Eens 1990).

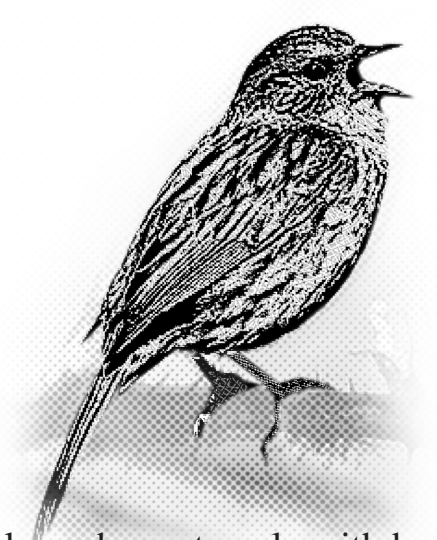

Polyandry. A single female mates with several males, who mate only with her. This rare mating system occurs, for example, in the Dunnock Prunella modularis, but polygyny and monogamy are also found in this species, depending on food resources (Davies and Lundberg 1984).

Polygynandry. In this, the most common breeding system, each sex mates with more than one member of the opposite sex. Polygynandry is frequent in mammals. While the offspring require intensive parental care (e.g., female mammals produce milk), the males provide no parental care in $95 \%$ of mammal species (CluttonBrock 1989).

females present in the population, $M\left(N_{m}, N_{f}\right)=N_{f}$. In this case, the two-sex model collapses to its one-sex, female counterpart. Figure 3.1 shows the shape of the limit function $\mu_{M}$ for more complex maing systems:

- For the monogamous mating system, males and females pair one-to-one and unpaired individuals do not reproduce. The mating function is $M\left(N_{m}, N_{f}\right)=$ $\min \left(N_{m}, N_{f}\right)$, and the corresponding limit function is $\mu_{M}(\sigma)=\min (1-\sigma, \sigma)$. This function has a tent shape with a single maximum at $\sigma=0.5$ (Figure 3.1a). - For the polygynous mating system with harem size $\theta$, a single male mates on average with $\theta$ females, giving $M\left(N_{m}, N_{f}\right)=\min \left(\theta N_{m}, N_{f}\right)$. The limit function is given by $\mu_{M}(\sigma)=\min (\theta(1-\sigma), \sigma)$; it has a maximum, as expected at $\sigma=\theta /(\theta+1)$, corresponding to one male mating with $\theta$ females (Figure 3.1b). 


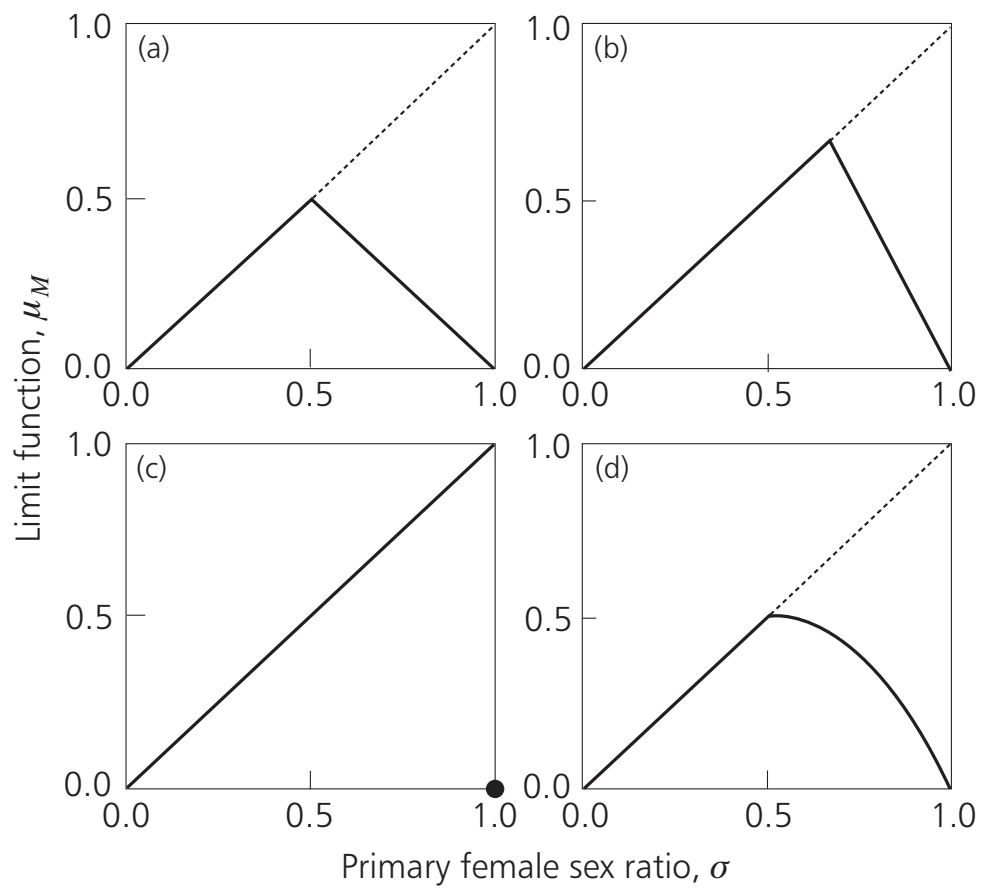

Figure 3.1 Limit functions for alternative mating systems. (a) Monogamy, (b) polygyny with harem size $\theta=2$, (c) polygyny with unrestricted harem size (the filled circle indicates that $\mu_{M}$ is discontinuous at $\sigma=1$, with $\mu_{M}(1)=0$ ), (d) harmonic-mean mating function.

For the polygynous mating system with unrestricted harem size, a single male can mate with as many females as he is willing to. The number of matings is equal to the number of females, except when there are no males, in which case the number of matings is 0 . The graph of the limit function coincides with the main diagonal except for $\sigma=1$, where $\mu_{M}$ is 0 (Figure 3.1c).

- The harmonic-mean mating function $M\left(N_{m}, N_{f}\right)=\min \left(2 N_{f} N_{m} /\left(N_{f}+N_{m}\right), N_{f}\right)$ can be seen as an intermediate pattern between monogamy and polygyny with a harem size of 2 . Indeed, each male mates on average with $2 \rho$ females, with $\rho$ being the breeding sex ratio. The graph of the limit function consists of a segment line and half of a parabola (Figure 3.1d).

Under rather general assumptions (the homogeneity property mentioned above being crucial), the two-sex model behaves as the one-sex model, with an asymptotic exponential growth and a stable population structure (Caswell and Weeks 1986; Martcheva 1999). As a result, the realized sex ratio (the proportion of females in the population) stabilizes, as does the breeding sex ratio. As with one-sex dynamics, two-sex dynamics can be decomposed into a transient regime followed by an asymptotic regime of exponential growth. However, convergence toward the equilibrium of the realized sex ratio interferes with convergence toward the stable age distribution, resulting in more complex transient dynamics than in one-sex models. Incorporating density dependence or competition between mates can generate even more complex population dynamics (Caswell and Weeks 1986; Chung 1994; Lindström and Kokko 1998). 


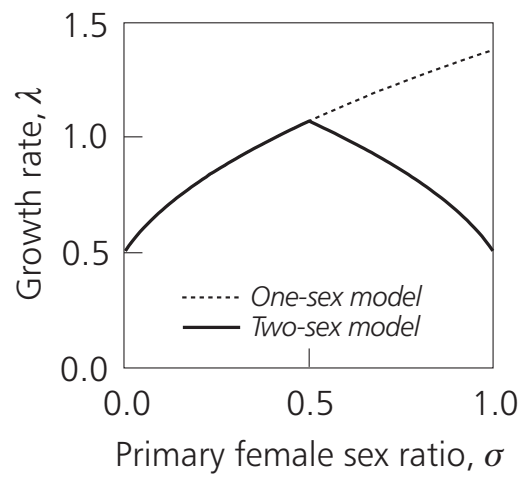

Figure 3.2 Growth rate as a function of the primary female sex ratio for the one-sex model and the two-sex model with monogamous mating function. The two curves coincide for $\sigma \leq 0.5$.

The two-sex expected growth rate, denoted by $\lambda_{M}$, depends on the mating function $M$ and on the male and female parts of the age-specific life cycle. Assuming that:

1. Males and females have identical survival rates,

2. Matings occur with equal probability among reproductive age classes,

3. A limit function $\mu_{M}$ can be associated to the mating function $M$ [Equation (3.10b)],

the growth rate $\lambda_{M}$ of the two-sex model with mating function $M$ satisfies (Legendre et al. 1999)

$$
\lambda_{M}(\sigma)=\lambda\left(\mu_{M}(\sigma)\right) .
$$

Thus, the two-sex growth rate is obtained by replacing the primary sex ratio $\sigma$ in the one-sex model with $\mu_{M}(\sigma)$. Since the one-sex growth rate $\lambda(\sigma)$ is a continuously increasing function of $\sigma$, Equation (3.10a) implies that the two-sex growth rate is always less than, or at most equal to, the one-sex growth rate, that is

$$
\lambda_{M}(\sigma) \leq \lambda(\sigma) .
$$

Thus, the maximum of the two-sex growth rate corresponds to the maximum of the limit function $\mu_{M}$ (compare Figure 3.1a with Figure 3.2).

For life cycles that are sex-symmetric, i.e., satisfying assumptions 1 and 2 above, the breeding sex ratio $\rho$ is equal to the primary sex ratio $\sigma$. However, sex-asymmetric life cycles exist in many species, often in relation to polygamy. In the polygynous mating system with harem size $\theta$, the optimal breeding sex ratio that maximizes the two-sex growth rate is $\rho_{\text {opt }}=\theta /(\theta+1)$, that is, $\theta$ females per male. Assuming a balanced primary sex ratio, $\sigma=0.5$, the optimal breeding sex ratio can be achieved by reducing the number of reproductive males in several nonexclusive ways:

- Males have lower (adult) survival rates,

- Males have delayed access to reproduction, 
Only a fraction of mature males have access to reproduction.

All three cases are known to occur in polygynous species.

\section{Influence of sexual reproduction on the extinction risk}

The effect of demographic stochasticity on the viability of a sexual, age-structured population can be investigated by constructing a branching process model based on the two-sex life cycle and the mating function (Harris 1963; Athreya and Ney 1972; Asmussen and Hering 1983; Gabriel and Bürger 1992; Kokko and Ebenhard 1996; Hull 1998; see also Chapter 2). Numbers of individuals that result from lifecycle transitions are drawn according to integer-valued probability distributions, depending on the number of individuals in age classes. For "all-or-nothing" transitions - such as surviving or not, being born as male or female, being reproductive or not - the number of individuals in the next age class is computed by summing Bernoulli trials (one trial per individual) or, equivalently, by sampling binomial distributions. For fecundity transitions, the number of offspring is computed as a sum of trials according to, for example, a Poisson distribution with a mean equal to the expected fecundity. For example, the two age-class matrix

$$
A=\left(\begin{array}{cc}
0 & \sigma B \\
\phi_{1} & \phi
\end{array}\right)
$$

leads to recursion Equations (3.12a) and (3.12b) from one time step to the next,

$$
\begin{aligned}
& N_{1}(t+1)=\operatorname{Poisson}(\sigma B) * N_{2}(t), \\
& N_{2}(t+1)=\operatorname{Binom}\left(N_{1}(t), \phi_{1}\right)+\operatorname{Binom}\left(N_{2}(t), \phi\right),
\end{aligned}
$$

where Poisson $(x) * N$ denotes the sum of $N$ samples of the Poisson distribution with mean $x$, and $\operatorname{Binom}(N, p)$ denotes the sum of $N$ Bernoulli trials of probability $p$. The corresponding two-sex model is specified by the equations

$$
\begin{aligned}
& J(t)=\operatorname{Poisson}(B) * M\left(N_{m 2}(t), N_{f 2}(t)\right), \\
& N_{f 1}(t+1)=\operatorname{Binom}(J(t), \sigma), \\
& N_{m 1}(t+1)=J(t)-N_{f 1}(t+1), \\
& N_{f 2}(t+1)=\operatorname{Binom}\left(N_{f 1}(t), \phi_{f 1}\right)+\operatorname{Binom}\left(N_{f 2}(t), \phi_{f}\right), \\
& N_{m 2}(t+1)=\operatorname{Binom}\left(N_{m 1}(t), \phi_{m 1}\right)+\operatorname{Binom}\left(N_{m 2}(t), \phi_{m}\right) .
\end{aligned}
$$

Equation (3.13a) gives the number $J$ of newborns produced according to the mating function $M$; Equations (3.13b) and (3.13c) split this number into males and females according to the primary female sex ratio $\sigma$; Equations (3.13d) and (3.13e) give the number of adult males and females that result from the survival of either subadult males and subadult females, or adult males and adult females. 
Under the assumptions 1 to 3 above, the two-sex population under demographic stochasticity either becomes extinct or grows on average at a rate $\lambda_{M}$, as in the one-sex case. This behavior seems general, but theoretical results are still lacking. Incorporation of both sexes and the mating system complicates the structure of the transient dynamics, and possibly reduces the long-term growth rate as a consequence. Thus, the two-sex branching process has a larger probability of extinction than the corresponding one-sex process, even when the two-sex growth rate is equal to the one-sex growth rate.

Extinction probabilities and the distribution of extinction time turn out to be highly dependent on the mating system. Real data - for passerines introduced to New Zealand (Legendre et al. 1999), and bighorn sheep in North America (see Section 3.4) - suggest that demographic stochasticity interacts with the mating system to determine the extinction risk of small populations. Long-lived and shortlived sexual species behave differently with regard to extinction because of the stochasticity of the mating process, one reason being that the elasticity of $\lambda$ to changes in the primary sex ratio $\sigma$ is inversely related to the generation time $\bar{T}$ [see Equation (3.5)]. Differentiating Equation (3.10a) with respect to $\sigma$ by the chain rule,

$$
\partial \lambda_{M} / \partial \sigma=\left(\partial \lambda / \partial \mu_{M}\right)\left(\partial \mu_{M} / \partial \sigma\right),
$$

shows that the sensitivity $\partial \lambda_{M} / \partial \sigma$ of the two-sex growth rate to changes in $\sigma$, and hence the probability of extinction, depends on the slope $\partial \mu_{M} / \partial \sigma$ of the limit function. As a result, the same growth rate can lead to different probabilities of extinction depending on the mating system. For example, the monogamous mating function and the harmonic-mean mating function produce the same two-sex growth rate when the primary sex ratio $\sigma$ is balanced, but the smoothness of the harmonic mean dramatically reduces the probability of extinction, as shown in Figure 3.3a. Furthermore, the value of $\sigma$ that maximizes the growth rate and the value of $\sigma$ that minimizes the probability of extinction usually differ. For the polygynous mating system with unrestricted harem size, the growth rate is maximized when the primary sex ratio $\sigma$ is close to 1 (Figure $3.1 \mathrm{c}$ ), but the probability of extinction shows a different pattern. If the proportion of females is low, few offspring are produced, and therefore the growth rate is less than 1 and extinction is certain. If the proportion of females is high, then the growth rate is large, but males can go extinct. The extinction risk turns out to be minimum for an intermediate value of $\sigma$, as shown in Figure 3.3b. Such contrasting effects of mating structure on a population's growth rate and extinction risk suggest that the adaptive evolution of sex-related life-history traits may have complex and unexpected effects on population viability.

\section{Sexual selection and extinction}

Males and females have conflicting interests in reproduction, and as a consequence natural selection operates differentially on each sex. This gives rise to sexual selection (Darwin 1871; Fisher 1958; Andersson 1994). A general pattern is that 
(a)

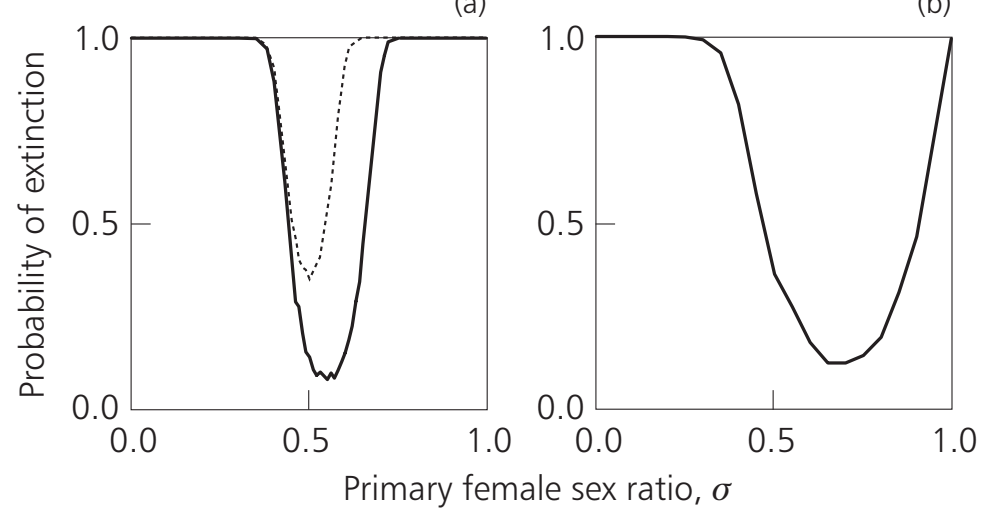

Figure 3.3 Probability of extinction as a function of the primary female sex ratio $\sigma$, for the two-sex model as specified in Section 3.3. (a) Monogamous mating system versus harmonic-mean mating function: the smoothness of the harmonic mean reduces the probability of extinction (dotted curve). The initial population comprised 20 adult males and 20 adult females. Monte Carlo simulations involved 1000 trials run over 100 time steps. (b) Polygynous mating system with unrestricted harem size: growth rate is maximal for $\sigma \approx 1$, while the probability of extinction is minimal for $\sigma \approx 0.66$. The initial population comprised five adult males and five adult females. Monte Carlo simulations involved 1000 trials run over 50 time steps. Parameters: $\phi_{1}=0.3, \phi=0.5, B=4.0$; growth rate $\lambda_{M}(\sigma)=1.064$ for $\sigma=0.5$.

females (with a small number of large gametes) are under selection to increase their reproductive success by searching for "good" males, while males can increase their fitness by copulating with many females (as males have a huge number of tiny motile gametes). This generally induces a larger variance in male reproductive success (Bateman 1948; Wade and Arnold 1980), and promotes sexual dimorphism, with the development of exaggerated ornaments, weapons, signals, or behaviors far beyond the expected optimal under the action of individual selection. In effect, sexually selected species seem more prone to extinction (McLain et al. 1995; Sorci et al. 1998). However, little theory deals with the impact of sexual selection on demography, as most models pertain to the field of population genetics (Lande 1980a; Kirkpatrick 1982; Pomiankowski et al. 1991; Iwasa et al. 1991) or to game theory (e.g., Maynard Smith 1982; Iwasa and Harada 1998), where, in both cases, demographic structure is usually ignored.

Under sexual selection, the evolution of male and female life-history traits may cause them to diverge, leading to sexual dimorphism. Sexual dimorphism on age at maturity (bimaturism) or survival can result in a strongly skewed breeding sex ratio. For example, in polygynous ungulates, adult males usually have lower survival rates than females; in the bighorn sheep, this difference yields a breeding sex ratio $\rho \approx 0.80$ (see Section 3.4). Results in this section suggest that sexual structure could have a significant impact on persistence. Could the individual behavior that determines the structure of a mating system evolve concomitantly with a reduced risk of extinction for the population? Evolutionary changes in sexual 
behavior may be tightly constrained, especially in species undergoing strong sexual selection. While the evolution of life histories has been studied in detail, very little is known of the effect of sexual reproduction and its evolution on population dynamics and viability.

\subsection{Interfacing Demography and Genetics}

In small populations, genetic drift (the genetic equivalent of demographic stochasticity) may beget the fixation of deleterious mutations (see Chapters 5 and 9). Assessing the impact of genetic drift on vital rates and population viability requires the development of integrated models that interface demography and genetics (Lande 1994, 1995; Mills and Smouse 1994; Lynch et al. 1995a). To this end, the approach followed by Thévenon and Couvet (2002), aims at tracking the population vector of age-class frequencies and the genetic vector of allelic frequencies simultaneously, while explicitly modeling interactions between the two. The so-called mutation load that results from deleterious mutations (see Chapter 9) affects the demographic parameters and therefore the population size and structure, which in turn modify the genetic composition of the population (Box 3.2). In

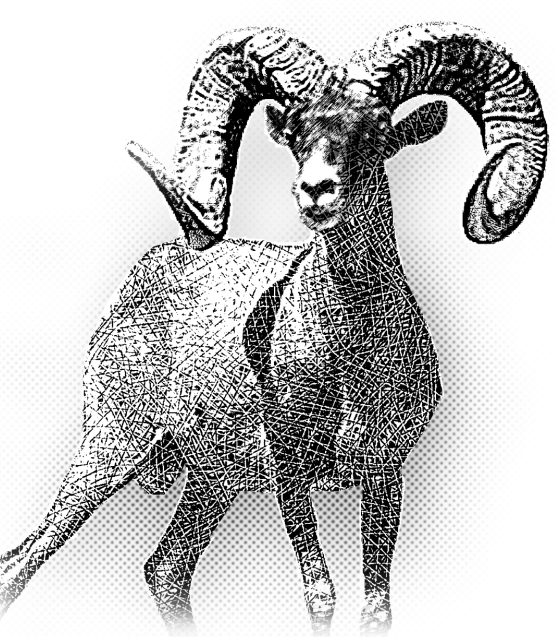

American bighorn sheep Ovis canadensis this approach, the effect of selection against deleterious mutation is accounted for (see Chapter 10). In their seminal work, Mills and Smouse (1994) also describe the combined effects of demography and genetics on the risk of population extinction, but selection was not part of their framework. Mildly deleterious mutations appear to be the most harmful, because, unlike strongly deleterious ones, they are not eliminated. Below, we illustrate these general considerations with a specific example, that of the dynamics of the American bighorn sheep, Ovis canadensis.

The American bighorn sheep of the Rocky mountains is a polygynous species that exhibits a strong sexual dimorphism. Females generally start to reproduce when 2-3 years old. Males mature when 3.5 years old on average, but competition between males usually means that they do not participate in reproduction until at an older age. Adult male survival rates are lower than those of females. Senescence starts after 7-8 years, and is more pronounced for males, but animals can live up to 20 years. The bighorn sheep model is summarized in Table 3.1.

Demographic parameters used in the model come from the literature (Geist 1971; Monson and Sumner 1981; Festa-Bianchet et al. 1995; Jorgenson et al. 1997). Females and males start to reproduce at 3 and 5 years of age, respectively. A polygynous mating system with harem size $\theta=4$ is used, matings being dispatched evenly between reproductive female age classes. The two-sex matrix is a 
Box 3.2 Inbreeding depression in structured population models

In a population, the fate of mutations depends on the initial allelic frequencies, the mutation rate $u$ (and reverse mutation rate $u_{\text {rev }}$ ), the selective pressures that eliminate deleterious genes, and genetic drift. For a given gene with wild and mutant alleles, we denote by $h$ the dominance of the deleterious allele, and by $s_{\text {repr }}$ and $s_{\text {surv }}$ the selection coefficients associated with reproduction and annual survival (juvenile survival or adult survival), respectively. For given frequencies $p$ and $q=1-p$ of the wild and mutant alleles, the expected frequency $q_{\mathrm{repr}}^{\prime}$ of the mutant allele after reproduction is

$$
q_{\mathrm{repr}}^{\prime}=\frac{p q\left(1-h s_{\mathrm{repr}}\right)+q^{2}\left(1-s_{\mathrm{repr}}\right)}{p^{2}+2 p q\left(1-h s_{\mathrm{repr}}\right)+q^{2}\left(1-s_{\mathrm{repr}}\right)}+\left(u p-u_{\mathrm{rev}} q\right) .
$$

After one time step, the expected frequency $q_{\text {surv }}^{\prime}$ of the mutant allele among surviving individuals is

$$
q_{\text {surv }}^{\prime}=\frac{p q\left(1-h s_{\text {surv }}\right)+q^{2}\left(1-s_{\text {surv }}\right)}{p^{2}+2 p q\left(1-h s_{\text {surv }}\right)+q^{2}\left(1-s_{\text {surv }}\right)} .
$$

The genetic composition of a population of $N$ individuals is described by a genetic vector $\Gamma$ with $2 N+1$ entries, in which the $k$ th entry gives the probability of a randomly chosen individual to have $k$ mutated genes. Through reproduction $N$ individuals produce $N^{\prime}$ offspring, among which the number of mutants is drawn from the binomial distribution $\operatorname{Binom}\left(2 N^{\prime}, q_{\mathrm{repr}}^{\prime}\right)$, with $q_{\mathrm{repr}}^{\prime}$ given by Equation (a). The resultant genetic vector $\Gamma^{\prime}=G_{\text {repr }}\left(\Gamma, N^{\prime}, s_{\text {repr }}\right)$ has $2 N^{\prime}+1$ entries. For survival, a hypergeometric distribution is used with expectation $q_{\mathrm{repr}}^{\prime}$, as given by Equation (b). The mutation load $G_{\text {load }}(\Gamma, s)$ that affects survival and fecundity parameters is computed according to

$$
G_{\text {load }}(\Gamma, s)=\prod_{k=0}^{2 N}\left[(1-h s)^{2 p q}(1-s)^{q^{2}}\right]^{n_{L} \Gamma_{k}},
$$

where $n_{L}$ denotes the number of loci, $\Gamma_{k}$ is the $k$ th entry of the genetic vector, and $s=s_{\text {repr }}$ or $s_{\text {surv }}$ depending on whether the affected trait is a fecundity parameter or a survival parameter. The genetic vectors $\Gamma^{(1)}$ and $\Gamma^{(2)}$ of two sets of individuals can be combined into a genetic vector $\Gamma=G_{\text {comp }}\left(\Gamma^{(1)}, \Gamma^{(2)}\right)$, where the operator $G_{\text {comp }}$ is such that the $k$ th entry of $\Gamma$ is given by

$$
\Gamma_{k}=\sum_{\substack{k_{1}, k_{2} \\ k_{1}+k_{2}=k}} \Gamma_{k_{1}}^{(1)} \Gamma_{k_{2}}^{(2)} .
$$

This associative composition can be extended to any number of genetic vectors.

For each age class $i$ containing $N_{i}$ individuals, there is a corresponding genetic vector $\Gamma^{(i)}$ with $2 N_{i}+1$ entries. Likewise, for each life-cycle transition $(i, j)$, there is an associated intermediate population size $N^{(i, j)}$ and an intermediate genetic vector $\Gamma^{(i, j)}$ with $2 N^{(i, j)}+1$ entries. From one time step to the next, the interactions of genetics and demography are incorporated in the one-sex two-age class matrix $\left(\begin{array}{cc}0 & \sigma B \\ \phi_{1} & \phi_{2}\end{array}\right)$ according to the following scheme: 


\section{Box 3.2 continued}

- Influence of genetics on demography. Compute the intermediate population sizes $N^{(i, j)}$, taking into account demographic stochasticity and mutational load,

$N^{\prime(2,1)}=\operatorname{Poisson}\left(G_{\text {load }}\left(\Gamma^{(2)}, s_{\text {repr }}\right) \times \sigma B\right) \times N_{2}$,

$N^{\prime(1,2)}=\operatorname{Binom}\left(N_{1}, G_{\text {load }}\left(\Gamma^{(1)}, s_{\text {surv }}\right) \times \phi_{1}\right)$,

$N^{\prime(2,2)}=\operatorname{Binom}\left(N_{2}, G_{\text {load }}\left(\Gamma^{(2)}, s_{\text {surv }}\right) \times \phi_{2}\right)$,

$N_{1}^{\prime}=N^{\prime(2,1)}$,

$N_{2}^{\prime}=N^{\prime(1,2)}+N^{\prime(2,2)}$.

Influence of demography on genetics. Update the intermediate genetic vectors $\Gamma^{(i, j)}$, taking the intermediate population sizes $N^{\prime(i, j)}$ into account, and compute the resultant genetic vectors $\Gamma^{\prime(j)}$,

$$
\begin{aligned}
& \Gamma^{\prime(1,2)}=G_{\text {surv }}\left(\Gamma^{(1)}, N^{\prime(1,2)}, s_{\text {surv }}\right) \\
& \Gamma^{\prime(2,2)}=G_{\text {surv }}\left(\Gamma^{(2)}, N^{\prime(2,2)}, s_{\text {surv }}\right) \\
& \Gamma^{\prime(2,1)}=G_{\text {repr }}\left(\Gamma^{(2)}, N^{\prime(2,1)}, s_{\text {repr }}\right) \\
& \Gamma^{\prime(1)}=\Gamma^{\prime(2,1)} \\
& \Gamma^{\prime(2)}=G_{\text {comp }}\left(\Gamma^{\prime(1,2)}, \Gamma^{\prime(2,2)}\right) .
\end{aligned}
$$

$14 \times 14$ block matrix with the upper diagonal block standing for the male life cycle, the lower diagonal block for the female life cycle, and the upper nondiagonal block for the production of male offspring by females.

The two-sex growth rate is $\lambda_{M}=1.03$. All female age classes have about the same reproductive value. The differences in male and female life cycles mean that the proportion of females in the population is $63 \%$. The breeding sex ratio is $\rho=0.81$, close to the optimum value $\rho_{\mathrm{opt}}=4 / 5=0.80$ (see Section 3.3). In fact, harem size has a significant impact on the growth rate, which underscores the importance of the mating system on population persistence. The effective population size, given by

$$
N_{e}=\frac{4 N_{m} N_{f}}{N_{f}+N_{m}}=4 N_{m} \rho,
$$

is equal to $36 \%$ of the total population size, close to the estimated value of $33 \%$ for bighorn populations in Wyoming (Fitzsimmons et al. 1997).

Demographic stochasticity is incorporated by treating by randomizing lifecycle transitions and matings as stochastic processes, as explained in Section 3.3. For simplicity, the initial populations are assumed to include individuals that belong to the oldest age class only, with $80 \%$ females and $20 \%$ males, close to the stable proportion given by the deterministic model. Probabilities of extinction are 
Table 3.1 Parameters used in the bighorn sheep population model.

\section{Demographic parameters}

Survival probabilities

Juvenile

$\phi_{m 0}$

Males

Females

Yearlings

$\phi_{m 1}$

0.57

$\phi_{f 0}$

0.57

Prime age

$\phi_{m 2}$

0.86

$\phi_{f 1}$

0.83

0.86

$\phi_{f 2}$

0.94

0.78

Older

$\phi_{m 3}, \phi_{m 4}, \phi_{m 5}, \phi_{m 6}$

0.63

$\phi_{f 3}, \phi_{f 4}, \phi_{f 5}, \phi_{f 6}$

$\phi_{f}$

0.85

Fecundities

$b_{3}, b_{4}, b_{5}, b_{6}, b_{7}$

0.70

\section{Population matrix}

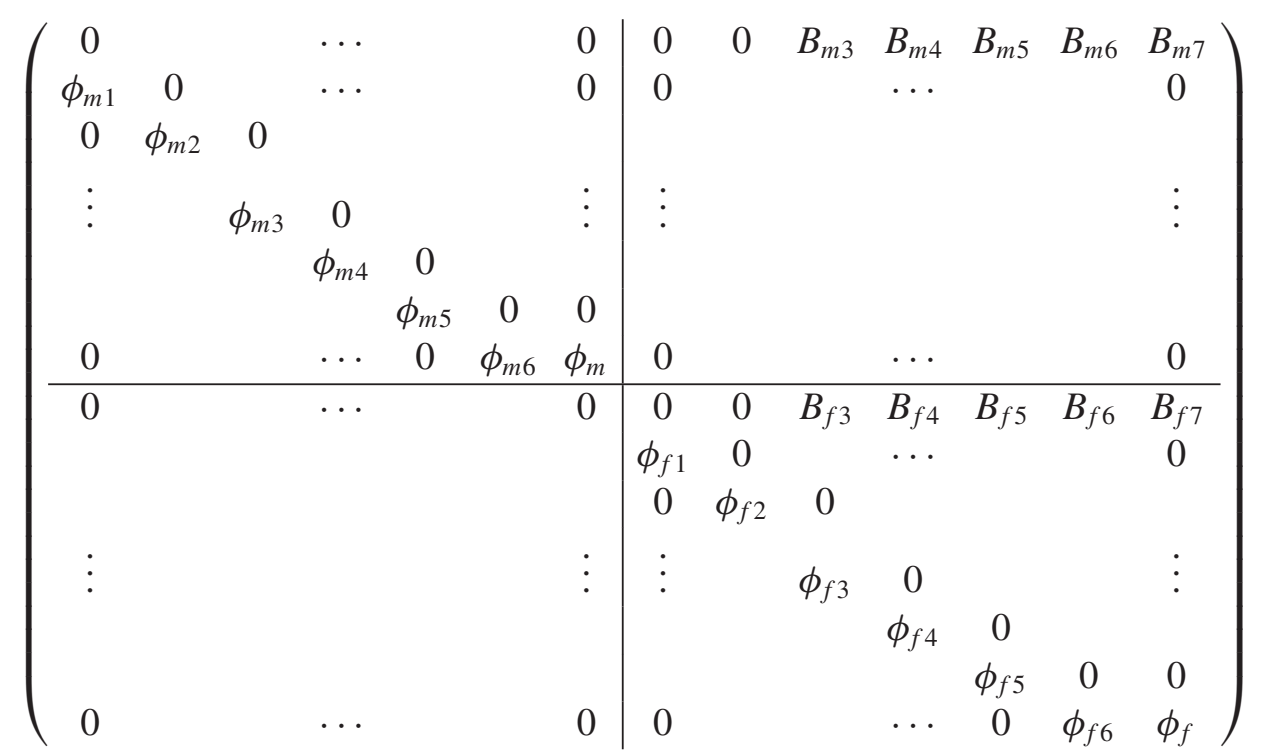

\section{Mating system}

Number of reproducing females: $N_{f}=N_{f 3}+N_{f 4}+N_{f 5}+N_{f 6}+N_{f 7}$

Number of reproducing males: $N_{m}=N_{m 5}+N_{m 6}+N_{m 7}$

Number of polygynous matings with harem size 4: $M=\min \left(4 N_{m}, N_{f}\right)$

Number of matings involving $i$-year-old females: $M_{i}=M N_{f i} / N_{f}$

Primary sex ratio: $\sigma=0.5$

Number of 1 -year-old males produced by $i$-year-old females: $B_{m i} N_{f i}=(1-\sigma) \phi_{m 0} b_{i} M_{i}$

Number of 1-year-old females produced by $i$-year-old females: $B_{f i} N_{f i}=\sigma \phi_{f 0} b_{i} M_{i}$

\section{Genetic parameters}

Dominance

Selection coefficient (reproduction)

Selection coefficient (annual survival)

Mutation rate

Reverse mutation rate

Number of loci

$$
\begin{aligned}
& h=0.2 \\
& s_{\text {repr }}=0.01 \\
& s_{\text {surv }}=0.0017 \\
& u=5 \quad 10^{-6} \\
& u_{\text {rev }}=5 \quad 10^{-7} \\
& n_{L}=10000
\end{aligned}
$$




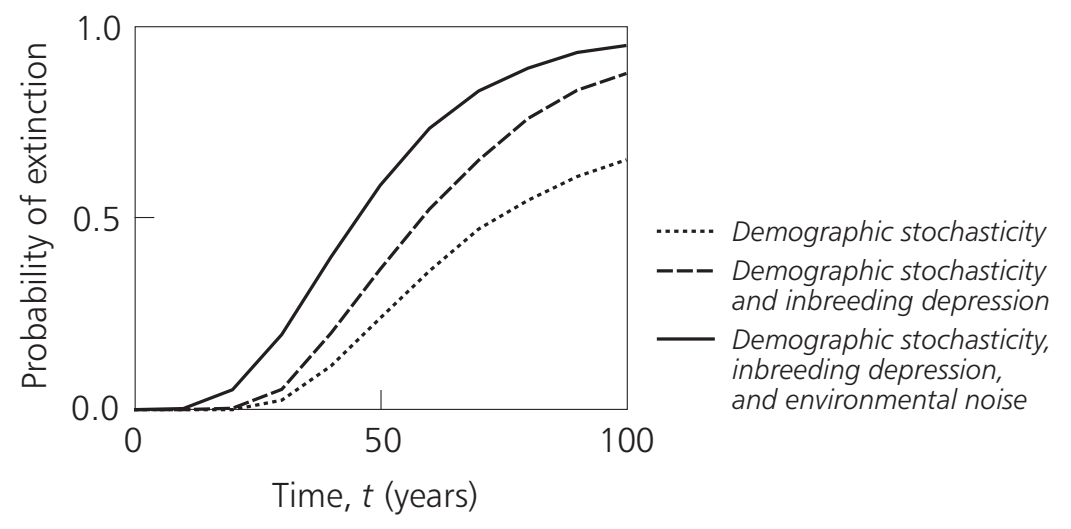

Figure 3.4 Probability of extinction, cumulated over time, as predicted by the bighorn sheep model. The initial population comprised 40 individuals; Monte Carlo simulations involved 1000 trials.
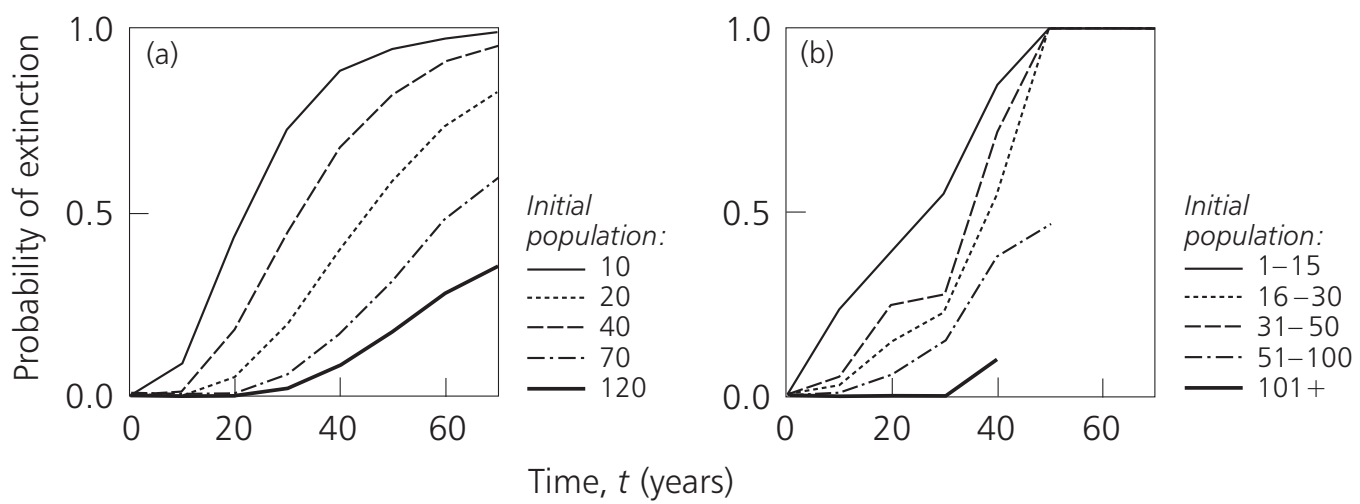

Figure 3.5 Probability of extinction, cumulated over time, for the bighorn sheep population. (a) Model results under the joint effects of demographic stochasticity, inbreeding depression, and environmental noise. Monte Carlo simulations involved 1000 trials. (b) Observed rates of extinction. Source: Berger (1990).

computed using Monte Carlo simulations that involve 1000 trials (Figures 3.4 and 3.5). Inbreeding depression was incorporated in the model as described in Box 3.2, with the parameters given in Table 3.1. Accounting for the genetic load induces a marked increase of extinction probability, as shown in Figure 3.4. Finally, environmental noise is incorporated in the model: demographic parameters vary from year to year according to probability beta distributions with means equal to estimated parameter values, and standard deviation fixed to 0.2. This extra noise further increases the probability of extinction (Figure 3.4). Demographic stochasticity, inbreeding depression, and environmental noise all have to be considered for the estimated probabilities of extinction to match the real data shown in Figure 3.5.

\subsection{Concluding Comments}

Selective pressures that affect phenotypes and the viability of a population both depend upon the structure of the individual life cycle. We have shown in this chapter that both the age structure and the mating system - two important characteristics 
of a species' life cycle - can have a dramatic impact on population viability. In a conservation perspective, population structure and sexual structure correspond to social bonds that management should take into account. For example, introducing adults is, in many cases, a better strategy than introducing young, immature individuals (Sarrazin and Legendre 2000), and sustainable hunting pressures depend on the breeding system (Greene et al. 1998; Wielgus et al. 2001).

Sensitivity analysis provides a powerful tool for estimating selection gradients that act on life-history traits (Lande 1982; Benton and Grant 1999). Parameters associated with higher sensitivities are under stronger selective pressures. In populations subject to environmental stochasticity, the resulting adaptive changes are expected to increase the deterministic growth rate and reduce the discount factor that accounts for environmental variation [see Equation (3.7)]. This is expected to reduce the extinction risk (Lande and Orzack 1988). The underlying theory, however, does not account for density-dependent mechanisms that result from interactions between individuals (see Chapters 2 and 11). Models that consider simultaneously the evolution of complex life cycles and population's extinction risk in a density-dependent context still need to be developed. One reason for the current lack of such models is that, traditionally, evolution has been conceived as an optimizing process, with the growth rate being maximized and the extinction risk being minimized as a putative consequence. Chapters in Part $\mathrm{C}$ point to alternative ways of thinking about the effect of life-history evolution on population dynamics.

Another aspect of individual responses to environmental change that must be expected to impact population viability is phenotypic plasticity. Very little attention has been paid so far to the effect of phenotypic plasticity on population dynamics. The analysis of an age-structured model of a population of Drosophila melanogaster contaminated by the $\mathrm{C}$ virus showed that the shortening of developmental time increases the growth rate, but could also increase the extinction risk (Thomas-Orillard and Legendre 1996). This example suggests that the plastic response of life histories to environmental threats may have contrasting and intricate effects on population dynamics and viability. Phenotypic plasticity may itself be adaptive, and study of the combined effects of plasticity and its evolution on population dynamics in changing environments offers a further challenge to eco-evolutionary theorists. 


\section{References}

References in the book in which this chapter is published are integrated in a single list, which appears on pp. 365-410. For the purpose of this reprint, references cited in the chapter have been assembled below.

Allen JC, Schaffer WM \& Rosko D (1993). Chaos reduces species extinction by amplifying local-population noise. Nature 364:229-232

Andersson M (1994). Sexual Selection. Princeton, NJ, USA: Princeton University Press

Arnold SJ \& Duvall D (1994). Animal mating systems: A synthesis based on selection theory. The American Naturalist 143:317-348

Asmussen S \& Hering H (1983). Branching Processes. Boston, MA, USA: Birkhäuser

Athreya KB \& Ney PE (1972). Branching Processes. New York, NY, USA: Springer-Verlag

Bateman AJ (1948). Intra-sexual selection in Drosophila. Heredity 2:349-368

Berger J (1990). Persistence of different-sized populations: An empirical assessment of rapid extinction in bighorn sheep. Conservation Biology 4:91-98

Castillo-Chavez C \& Hsu Schmitz S-F (1997). The evolution of age-structured marriage functions: It takes two to tango. In Structured-population Models in Marine, Terrestrial and Freshwater Systems, eds. Tuljapurkar S \& Caswell H, pp. 533-553. New York, NY, USA: Chapman \& Hall

Caswell H (1989). Matrix Population Models. Sunderland, MA, USA: Sinauer Associates Inc.

Caswell H (2001). Matrix Population Models, Second Edition. Sunderland, MA, USA: Sinauer Associates Inc.

Caswell H \& Weeks DE (1986). Two-sex models: Chaos, extinction, and other dynamic consequences of sex. The American Naturalist 128:707-735

Chung R (1994). Cycles in the two-sex problem: An investigation of a nonlinear demographic model. Mathematical Population Studies 5:45-73

Clutton-Brock TH (1989). Mammalian mating systems. Proceedings of the Royal Society of London B 236:339-372

Courchamp F, Clutton-Brock T \& Grenfell B (1999). Inverse density dependence and the Allee effect. Trends in Ecology and Evolution 14:405-410

Darwin C (1871). The Descent of Man and Selection in Relation to Sex. London, UK: J. Murray

Davies NB \& Lundberg A (1984). Food distribution and a variable mating system in the dunnock (Prunella modularis). Journal of Animal Ecology 53:895-912

Ferrière R \& Gatto M (1993). Chaotic population dynamics can result from natural selection. Proceedings of the Royal Society of London B 251:33-38

Ferrière R, Sarrazin F, Legendre S \& Baron J-P (1996). Matrix population models applied to viability analysis and conservation: Theory and practice using the ULM software. Acta Oecologica 17:629-656

Festa-Bianchet M, Jorgenson JT, Lucherini M \& Wishart WD (1995). Life history consequences of variation in age of primiparity in bighorn ewes. Ecology 76:871-881

Fieberg J \& Ellner SP (2001). Stochastic matrix models for conservation and management: A comparative review of methods. Ecology Letters 4:244-266

Fisher RA (1958). The Genetical Theory of Natural Selection. New York, NY, USA: Dover Publications

Fitzsimmons NN, Buskirk SW \& Smith MH (1997). Genetic changes in reintroduced rocky mountain bighorn sheep populations. Journal of Wildlife Management 61:863-872 
Gabriel W \& Bürger R (1992). Survival of small populations under demographic stochasticity. Theoretical Population Biology 41:44-71

Geist V (1971). Mountain Sheep. Chicago, IL, USA: University of Chicago Press

Gerritsen J (1980). Sex and parthenogenesis in sparse populations. The American Naturalist 115:718-742

Gimelfarb A (1988). Processes of pair formation leading to assortative mating in biological populations: Encounter-mating model. The American Naturalist 131:865-884

Gosselin F \& Lebreton JD (2000). Potential of branching processes as a modeling tool for conservation biology. In Quantitative Methods for Conservation Biology, eds. Ferson S \& Burgman M, pp. 199-225. New York, NY, USA: Springer-Verlag

Greene C, Umbanhowar J, Mangel M \& Caro T (1998). Animal breeding systems, hunter selectivity, and consumptive use in wildlife conservation. In Behavioral Ecology and Conservation Biology, ed. Caro T, pp. 271-305. New York, NY, USA: Oxford University Press

Griffith B, Scott MJ, Carpenter JW \& Reed C (1989). Translocation as a species conservation tool: Status and strategy. Science 245:477-480

Harris TE (1963). The Theory of Branching Processes. Berlin, Germany: Springer-Verlag

Heino M, Metz JAJ \& Kaitala V (1998). The enigma of frequency-dependent selection. Trends in Ecology and Evolution 13:367-370

Höglund J (1996). Can mating systems affect local extinction risks? Two examples of lek-breeding waders. Oikos 77:184-188

Houllier F \& Lebreton J-D (1986). A renewal equation approach to the dynamics of stage grouped populations. Mathematical Biosciences 79:185-197

Hull DM (1998). A reconsideration of Galton's problem (using a two-sex population). Theoretical Population Biology 54:105-116

Iwasa Y \& Harada Y (1998). Female mate preference to maximize paternal care. II. Female competition leads to monogamy. The American Naturalist 151:367-382

Iwasa Y, Pomiankowski A \& Nee S (1991). The evolution of costly mate preferences. II. The "handicap" principle. Evolution 45:1431-1442

Jorgenson JT, Festa-Bianchet M, Gaillard J-M \& Wishart WD (1997). Effects of age, sex, disease, and density on survival of bighorn sheep. Ecology 78:1019-1032

Kirkpatrick M (1982). Sexual selection and the evolution of female choice. Evolution 36:1-12

Kokko H \& Ebenhard T (1996). Measuring the strength of demographic stochasticity. Journal of Theoretical Biology 183:169-178

Lande R (1980a). Sexual dimorphism, sexual selection, and adaptation in polygenic characters. Evolution 34:292-305

Lande R (1982). A quantitative genetic theory of life-history evolution. Ecology 63:607615

Lande R (1993). Risks of population extinction from demographic and environmental stochasticity and random catastrophes. The American Naturalist 142:911-927

Lande R (1994). Risk of population extinction from fixation of new deleterious mutations. Evolution 48:1460-1469

Lande R (1995). Mutation and conservation. Conservation Biology 9:782-791

Lande R \& Orzack S (1988). Extinction dynamics of age-structured populations in a fluctuating environment. Proceedings of the National Academy of Sciences of the USA 85:7418-7421 
Legendre S, Clobert J, Møller AP \& Sorci G (1999). Demographic stochasticity and social mating system in the process of extinction of small populations: The case of passerines introduced to New Zealand. The American Naturalist 153:449-463

Leirs H, Stenseth NC, Nichols JD, Hines JE, Verhagen R \& Verheyen W (1997). Stochastic seasonality and nonlinear density-dependent factors regulate population size in an African rodent. Nature 389:176-180

Lindström J \& Kokko H (1998). Sexual reproduction and population dynamics: The role of polygyny and demographic sex differences. Proceedings of the Royal Society of London B 265:483-488

Lynch M, Conery J \& Bürger R (1995a). Mutation accumulation and the extinction of small populations. The American Naturalist 146:489-518

Martcheva M (1999). Exponential growth in age-structured two-sex populations. Mathematical Biosciences 157:1-22

May RM \& Oster GF (1976). Bifurcations and dynamic complexity in simple ecological models. The American Naturalist 110:573-599

Maynard Smith J (1982). Evolution and the Theory of Games. Cambridge, UK: Cambridge University Press

McLain DK, Moulton MP \& Redfearn TP (1995). Sexual selection and the risk of extinction of introduced birds on oceanic islands. Oikos 74:27-34

Mills LS \& Smouse PE (1994). Demographic consequences of inbreeding in remnant populations. The American Naturalist 144:412-431

Mills LS, Hayes SG, Baldwin C, Wisdon MJ, Citta J, Mattson DJ \& Murphy K (1996). Factors leading to different viability predictions for a grizzly bear data set. Conservation Biology 10:863-873

Mills LS, Doak DF \& Wisdom MJ (1999). Reliability of conservation actions based on elasticity analysis of matrix models. Conservation Biology 13:815-829

Møller AP \& Birkhead TR (1994). The evolution of plumage brightness in birds is related to extrapair paternity. Evolution 48:1089-1100

Møller AP \& Legendre S (2001). Allee effect, sexual selection and demographic stochasticity. Oikos 92:27-34

Monson G \& Sumner L (1981). The Desert Bighorn. Tucson, AZ, USA: The University of Arizona Press

Orians G (1969). On the evolution of mating systems in birds and mammals. The American Naturalist 103:589-603

Pinxten R \& Eens M (1990). Polygyny in the European starling: Effect of female reproductive success. Animal Behaviour 40:1035-1047

Pomiankowski A, Isawa Y \& Nee S (1991). The evolution of costly mate preferences. I. Fisher and biased mutation. Evolution 45:1422-1430

Ruelle D (1989). Chaotic Evolution and Strange Attractor. Cambridge, UK: Cambridge University Press

Sarrazin F \& Legendre S (2000). Demographic approach to releasing adults versus young in reintroduction. Conservation Biology 14:474-487

Shaffer M (1987). Minimum viable populations: Coping with uncertainty. In Viable Populations for Conservation, ed. Soulé ME, pp. 87-123. Cambridge, UK: Cambridge University Press

Sorci G, Møller AP \& Clobert J (1998). Plumage dichromatism predicts introduction success in New Zealand. Journal of Animal Ecology 67:263-269

Stearns SC (1992). The Evolution of Life Histories. Oxford, UK: Oxford University Press 
Stephens PA \& Sutherland WJ (1999). Consequences of the Allee effect for behaviour, ecology and conservation. Trends in Ecology and Evolution 14:401-405

Stephens PA, Sutherland WJ \& Freckleton RP (1999). What is the Allee effect? Oikos 87:185-190

Thévenon S \& Couvet D (2002). The impact of inbreeding depression on population survival depending on demographic parameters. Animal Conservation 5:53-60

Thomas-Orillard M \& Legendre S (1996). Drosophila C virus and host-population dynamics. Comptes Rendus de l'Académie des Sciences de Paris 319:615-621

Tuljapurkar S (1990). Population Dynamics in Variable Environments. Berlin, Germany: Springer-Verlag

Wade MJ \& Arnold SJ (1980). The intensity of sexual selection in relation to male sexual behaviour, female choice, and sperm precedence. Animal Behaviour 28:446-461 